\title{
The thermal emission of the exoplanet WASP-3b
}

\author{
John W. Rostron, ${ }^{1 \star}$ Peter J. Wheatley, ${ }^{1}$ David R. Anderson, ${ }^{2}$ \\ Andrew Collier Cameron, ${ }^{3}$ Jonathan J. Fortney, ${ }^{4}$ Joseph Harrington, ${ }^{5}$ \\ Heather A. Knutson ${ }^{6}$ and Don L. Pollacco ${ }^{1}$ \\ ${ }^{1}$ Department of Physics, University of Warwick, Coventry CV4 7AL, UK \\ ${ }^{2}$ Astrophysics Group, Keele University, Staffordshire ST5 5BG, UK \\ ${ }^{3}$ School of Physics and Astronomy, University of St Andrews, St Andrews, Fife KY16 9SS, UK \\ ${ }^{4}$ Department of Astronomy and Astrophysics, University of California, Santa Cruz, CA 95064, USA \\ ${ }^{5}$ Planetary Sciences Group, Department of Physics, University of Central Florida, Orlando, FL 32816-2385, USA \\ ${ }^{6}$ Division of Geological and Planetary Sciences, California Institute of Technology, Pasadena, CA 91125, USA
}

Accepted 2014 April 23. Received 2014 April 14; in original form 2014 January 19

\begin{abstract}
We report the detection of thermal emission from the transiting hot Jupiter WASP-3b at 3.6, 4.5 and $8.0 \mu \mathrm{m}$ using the Spitzer Space Telescope. We obtain planet-to-star flux ratios of $0.209_{-0.028}^{+0.040}, 0.282 \pm 0.012$ and $0.328_{-0.055}^{+0.086}$ percent at these wavelengths, respectively, implying infrared brightness temperatures of $T_{3.6 \mu \mathrm{m}}=2280_{-150}^{+210} \mathrm{~K}, T_{4.5 \mu \mathrm{m}}=2400 \pm 80 \mathrm{~K}$ and $T_{8.0 \mu \mathrm{m}}=2210_{-250}^{+390} \mathrm{~K}$. We find that WASP-3b falls into an emerging class of highly irradiated planets whose measured temperatures suggest that the planets are dark and redistribute heat around the planet inefficiently. The latter is similarly concluded from 1D atmospheric model comparisons, which also favour the presence of an atmospheric temperature inversion. We compare the WASP-3 system to the proposed inversion-activity relation, finding that it hints at a more complex relation than a simple cut-off in activity implied by previous data. Using eclipse timings we also constrain $e \cos \omega$ to be $-0.0006_{-0.0006}^{+0.0010}$, suggesting that the eccentricity of WASP-3b can only be large for a narrow range of $\omega$.
\end{abstract}

Key words: methods: data analysis - planets and satellites: atmospheres - planets and satellites: individual: WASP-3b-stars: individual: WASP-3.

\section{INTRODUCTION}

Photometric and spectroscopic observations of transiting exoplanetary systems can be used to characterize planetary masses, radii, orbital and atmospheric properties (e.g. Charbonneau et al. 2000, 2002; Mazeh et al. 2000; Deming et al. 2005). One avenue for studying the latter is through observations of the system's secondary eclipse (where the planet passes behind the host star). Multiwavelength infrared observations of this kind probe the day-side thermal spectrum of the planet and can be used to constrain molecular compositions and vertical temperature structures (Sudarsky, Burrows \& Hubeny 2003; Burrows, Hubeny \& Sudarsky 2005; Fortney et al. 2005, 2008; Knutson et al. 2008). Complementary phase curve observations, which give planetary emission as a function of longitude, and transmission spectra, which sample absorption on the planetary limb, can further reveal information on abundances, albedos and heat transport efficiencies (Charbonneau et al. 2002; Barman, Hauschildt \& Allard 2005; Burrows, Sudarsky \& Hubeny 2006; Knutson et al. 2007).

\footnotetext{
^E-mail: J.W.Rostron@warwick.ac.uk
}

For most systems, secondary eclipse observations are currently only available in a few broad-band wavelength ranges. Whilst useful for gaining insights such as in discerning different temperature structures (e.g. temperature inversions; Knutson et al. 2008), potential ambiguities with chemical abundance ratios have also been highlighted (Madhusudhan \& Seager 2010).

Temperature inversions, where temperature increases with altitude, were first suggested for hot Jupiters by Hubeny, Burrows \& Sudarsky (2003). The first observational evidence was given by Knutson et al. (2008) for HD 209458b. Spitzer/Infrared Array Camera (IRAC) secondary eclipse observations revealed planetary spectral emission features of $\mathrm{H}_{2} \mathrm{O}$ and $\mathrm{CO}_{2}$, rather than the expected corresponding absorption features, as were found for example for HD 189733b (Charbonneau et al. 2008). Further temperature inversion characterisations have been made for other planets using Spitzer/IRAC secondary eclipse observations (e.g. Knutson, Howard \& Isaacson 2010, and references therein) with examples of both inverted and non-inverted atmospheres being found.

Fortney et al. (2008) proposed that hot Jupiters could fall into two classes, with the upper atmospheres of very hot planets being driven into emission due to the presence of gaseous $\mathrm{TiO}$ and VO. In less highly irradiated planets, these gases would condense out of the 
atmosphere and so would not drive an inversion. However, a number of planets have been found to contradict this (e.g. Machalek et al. 2008; Fressin et al. 2010), and searches for TiO in the transmission spectra of highly irradiated planets have shown little evidence for its presence (Huitson et al. 2013; Sing et al. 2013). The effects of other potential opacity sources have been explored, for example sulphur compounds (Zahnle et al. 2009), but the observed split in temperature structures remains unexplained.

Knutson et al. (2010) proposed a new mechanism to explain the presence or absence of temperature inversions, based on the activity of the host star. Under this mechanism, an as yet unknown absorber drives an atmospheric inversion except in the case of planets orbiting active stars, where the heightened UV flux breaks up the absorber, suppressing the inversion. Subsequent observations appear to be consistent with this idea (e.g. Anderson et al. 2013), though the correlation between host star activity and the nature of the inversion remains somewhat tentative.

In addition to distinguishing vertical temperature structures, secondary eclipse and phase curve observations have been used to constrain Bond albedos and heat transport efficiencies for shortperiod exoplanets (Cowan \& Agol 2011). Interestingly, Cowan \& Agol (2011) have found some evidence for an emerging population of very hot planets that have low albedos and low recirculation efficiencies.

\subsection{WASP-3}

WASP-3b (Pollacco et al. 2008) is a $1.4 R_{\mathrm{J}}$ planet orbiting a late F-type star at a separation of 0.03 au. The intense irradiation the planet experiences $\left(T_{\mathrm{eq}}=1960_{-76}^{+33} \mathrm{~K}\right.$; Pollacco et al. 2008) makes it an excellent target for studies of its thermal emission. A groundbased secondary eclipse observation in the $K_{\mathrm{s}}$ band has already been carried out, by Zhao et al. (2012). The brightness temperature derived from the eclipse measurement is around $2700 \mathrm{~K}$ - larger than the maximum expected from simple equilibrium temperature calculations. The Spitzer/IRAC secondary eclipse observations of WASP-3 presented in this paper reveal the planet's thermal emission over a wider range of infrared wavelengths.

With regard to the activity-inversion correlation, the activity measure for WASP-3 $\left(\log R_{\mathrm{HK}}^{\prime}=-4.872\right.$; Knutson et al. 2010) puts it interestingly near the expected cut-off between inverted and non-inverted atmospheres $\left(\log R_{\mathrm{HK}}^{\prime} \sim-4.9\right)$. A conclusive determination of the nature of WASP-3b's inversion would be useful in characterizing this cut-off. Equilibrium temperature estimates also suggest that WASP-3b could be part of the group of planets with low albedo and heat redistribution efficiencies highlighted by Cowan \& Agol (2011). Accurate brightness temperature estimates across the IRAC bands allow us to test this hypothesis.

We order the remainder of the paper as follows: in Section 2 we describe the secondary eclipse observations taken using Spitzer; the analysis carried out on these observations, including light curve model fitting, is described in Section 3; in Section 4 we present the results of this analysis; and in Section 5 these results are discussed in the context described above. Finally, we summarize our findings in Section 6.

\section{OBSERVATIONS}

During its cryogenic mission, the Spitzer Space Telescope's IRAC (Hora et al. 2004) provided imaging in four wavelength bands, centred on 3.6, 4.5, 5.8 and $8.0 \mu \mathrm{m}$ (channels 1-4, respectively). In mid-2009 Spitzer exhausted its liquid helium cryogen and be- gan the on-going warm mission, during which only the 3.6 and $4.5 \mu \mathrm{m}$ IRAC channels are available. The 5.8 and $8.0 \mu \mathrm{m}$ channels are unusable due to the heightened thermal background. It has been demonstrated, however, that the two remaining channels can still provide useful information on the presence or absence of temperature inversions in hot Jupiters (Knutson et al. 2010).

Observations of WASP-3 were taken at the predicted time of secondary eclipse on 2008 September 18, during the cryogenic mission. Simultaneous exposures of $12 \mathrm{~s}$ (effective integration time of $10.4 \mathrm{~s}$ ) were taken in full-array mode at 4.5 and $8.0 \mu \mathrm{m}$ over a period of $5.8 \mathrm{~h}$, providing 1559 images in each channel (programme ID 50759, PI: P. Wheatley).

Secondary eclipse measurements were also made at $3.6 \mu \mathrm{m}$ on 2009 October 26, during the warm mission. Observations were taken using IRAC's sub-array mode with a frame time of $2 \mathrm{~s}(1.92 \mathrm{~s}$ effective exposure), over $7.7 \mathrm{~h}$, providing 13670 individual images (programme ID 60021, PI: H. Knutson). The combination of the $2 \mathrm{~s}$ frame time and sub-array mode provided the best sensitivity whilst avoiding saturation of the star. The short exposures were also useful in the removal of systematics from the data.

\section{ANALYSIS}

\subsection{Basic Calibrated Data}

To analyse these data, we used Basic Calibrated Data (BCD) frames produced by the IRAC Level 1 pipeline (version S18.18.0), downloaded from the Spitzer Heritage Archive. ${ }^{1}$ These frames are flux calibrated, with dark and flat-field corrections applied and various well-known instrumental signatures mitigated. ${ }^{2}$ Before obtaining the desired flux measurements from these frames, we applied additional modifications, which are described below for each channel.

\subsubsection{Channel $1(3.6 \mu \mathrm{m})$ sub-array warm mission data}

IRAC sub-array data are provided in the form of data cubes, each containing $6432 \times 32$ pixel frames. 215 data cubes were obtained from the channel 1 observations. We elected to split each cube into individual images after assessing the autocorrelation of WASP-3's image centroid. The $y$-centroid autocorrelation function is shown in Fig. 1, where two clear features are evident. First, there is a long time-scale $(\sim 1 \mathrm{~h})$ variation corresponding to Spitzer's pointing wobble. ${ }^{3}$ This variation is also apparent in the raw photometry for channel 1 (upper-left plot of Fig. 4). Secondly, a sharp peak in the autocorrelation function is seen at small offsets. The width of this peak implies that short-time-scale excursions of the image centroid are present and typically last for $\sim 20 \mathrm{~s}$. Given that the duration of our data cubes was $\sim 120 \mathrm{~s}$, we felt that summing our frames, to produce one image per data cube, would not sufficiently sample this short-time-scale variation.

We used timing information found in the BCD FITS file headers to determine the Heliocentric Modified Julian Date (HMJD) times for each individual frame at mid-exposure. We also converted the times from the UTC to the terrestrial timing standard (TT) for consistency with the ephemeris we used to constrain our parameter fits (see Section 3.4).

\footnotetext{
${ }^{1}$ http://irsa.ipac.caltech.edu/data/SPITZER/docs/spitzerdataarchives/

${ }^{2}$ http://irsa.ipac.caltech.edu/data/SPITZER/docs/irac/ iracinstrumenthandbook/

${ }^{3}$ ssc.spitzer.caltech.edu/warmmission/news/21oct2010memo.pdf
} 


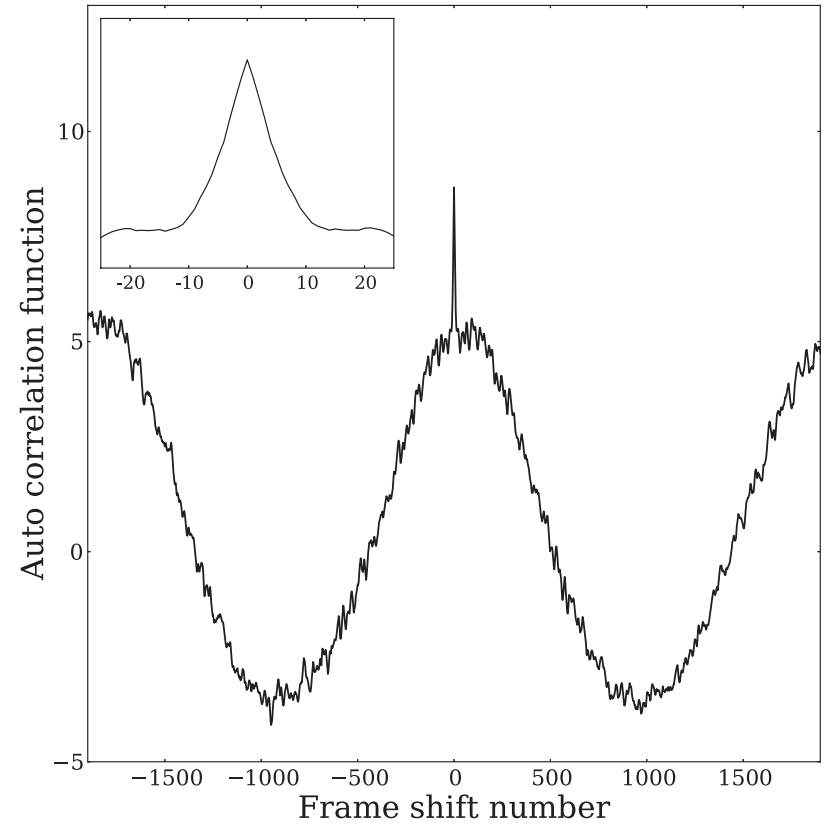

Figure 1. Autocorrelation of the $y$-centroid position of WASP-3 in the channel 1 sub-array images. Only the central 2000 images were used for the comparison time series. The long time-scale $(\sim 1 \mathrm{~h})$ variation is due to Spitzer's pointing wobble, which is also evident in the extracted flux values - see Fig. 4. The width of the central peak (see the inset, zoomed plot) suggests that shorter time-scale excursions of the image centroid typically last $\sim 20 \mathrm{~s}$. Similar trends are found for WASP-3's $x$-centroid position and flux time series.

In order to estimate errors on the flux values, we converted pixel values from $\mathrm{MJy} \mathrm{sr}^{-1}$ (as given for the $\mathrm{BCD}$ frames) to electron units by multiplying by the gain and effective exposure time and dividing by a flux conversion factor. These values were all taken from the FITS file headers. An estimate for the zodiacal background flux in the subtracted sky dark was also added, using the SKYDRKZB FITS header value (Anderson et al. 2011).

We found a trend in background values repeated in each data cube, similar to that seen in other IRAC data (e.g. Harrington et al. 2007; Agol et al. 2010; Deming et al. 2011; Todorov et al. 2012, 2013) - see Fig. 2. The 1st and 58th frames within each cube are clearly the largest outliers. The same trend is no longer seen for background-subtracted source fluxes, although the first frame in each cube still remains an outlier. Because of this, we chose to remove the first frame in each data cube for the remainder of the analysis. 215 (1.6 per cent) of the channel 1 frames were removed here.

\subsubsection{Channel $2(4.5 \mu \mathrm{m})$ cryogenic mission data}

Pixel values in channel 2 were converted from $\mathrm{MJy} \mathrm{sr}^{-1}$ to electrons in the same way as described in channel 1. FITS header timing information was again used to determine the $\operatorname{HMJD}(\mathrm{TT})$ times at mid-exposure for each frame.

We found that a small number of the channel 2 frames were affected by the 'column pull-down' effect. ${ }^{2}$ This causes a change in the intensity of pixels in the same column as very bright sources such as saturated stars and cosmic ray hits. There are no saturated stars in our frames, but cosmic ray hits do cause temporary changes to column intensity values. In addition to the 'pull-down', we also

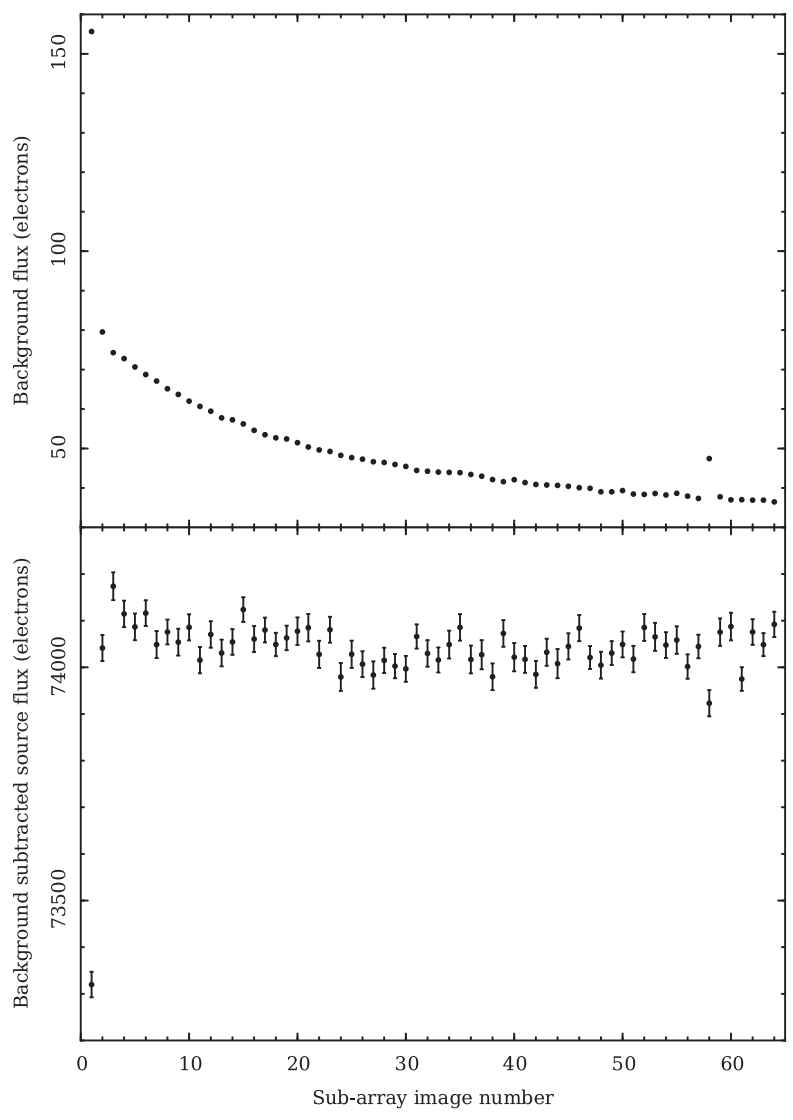

Figure 2. Upper panel: channel 1 clipped mean background values for a given sub-array image within each data cube are plotted. For each sub-array image, the average value across the 215 cubes is given here. A clear trend is seen with background values decreasing asymptotically. The 1st and 58th frames stand out as being discrepant from this trend, as has been found in other channel 1 warm mission data (e.g. Deming et al. 2011). The errors on these values are smaller than the plotted points. Lower panel: as above, except here we plot the background subtracted source flux (using a source aperture of 2.5 pixels centred on WASP-3). Here only the first frame is an outlier (at $14 \sigma$ ) and so the first frame in each data cube is rejected. The 58th frame is $2.5 \sigma$ from the median of the 64 values plotted. We elect to use these frames, since the background subtraction adequately accounts for the effect seen in the upper panel.

see a rise in the intensity of column values in frames directly after those affected by cosmic ray hits. We remove frames that show signs of these effects by creating time series of median column values and rejecting frames where any column is more than $10 \sigma$ away from the median-filtered time series for that column (using a window width of 20 observations). We applied this only to columns coincident with the source aperture, trusting that for the background estimate our clipped mean procedure (see Section 3.2) would account for this effect. For our optimal source aperture size of 3 pixels, we removed three frames as a result of this effect.

Post-science blank sky observations were carried out to check for warm pixels that could compromise our analysis of WASP-3. We found no evidence for such warm pixels.

\subsubsection{Channel $4(8.0 \mu \mathrm{m})$ cryogenic mission data}

Electron pixel values and mid-exposure HMJD(TT) times for channel 4 were calculated as for channel 2 . 

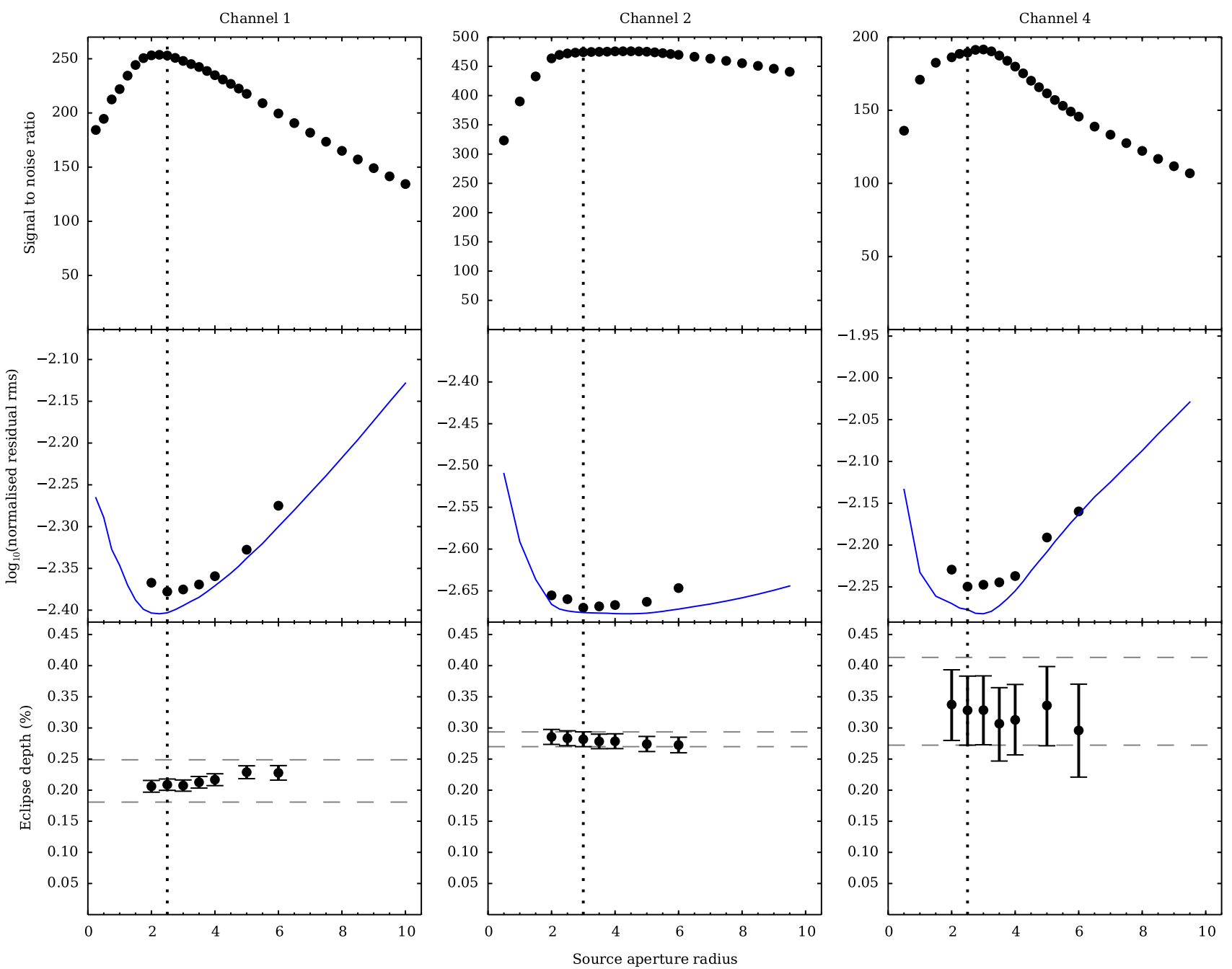

Figure 3. Columns from left to right are for channels 1, 2 and 4, respectively. The top row shows signal-to-noise ratios of flux measurements from a typical image of WASP-3, at a range of source aperture radii, with flux and error values determined as described in the text. The middle row gives normalized residual rms values on the detrended light curves, as a function of source aperture radius (black points). Also shown is the expectation from the signal-to-noise values (solid blue line). A discrepancy between these values, for a given source aperture radius, suggests the presence of additional noise sources. The bottom row gives the eclipse depth values, with MCMC errors. The grey dashed lines show the final quoted error for our optimal eclipse depth, taken as either the error from the MCMC or the prayer bead analysis, whichever was largest. The vertical dotted lines show the adopted radii, chosen to be where the normalized residual rms value was minimized.

Again, a small number of frames were affected by detector effects - this time by 'row pull-up', a result of electronic banding ${ }^{2}$ caused by cosmic ray hits. We removed affected frames in an equivalent way to that described for the 'column pull-down' effect in channel 2, but this time by creating time series of median row values. For our optimal source aperture size of 2.5 pixels, four frames were removed in this way.

As for channel 2, post-science blank sky observations were carried out. Again, no evidence of warm pixels that would affect our analysis of WASP-3 was found.

\subsection{Aperture photometry}

Simple aperture photometry was performed on the remaining frames using the ULTRACAM pipeline software (Dhillon et al. 2007). A range of source aperture radii were tested, from 2.0 to 4.0 pixels in steps of 0.5 pixels and additionally 5 and 6 pixel radii. In each channel, our adopted radius was selected on the basis that it minimized the normalized rms of the residuals on the final light curve. The final values adopted were 2.5, 3.0 and 2.5 pixels for channels 1 , 2 and 4, respectively (see Sections 3.7 and 3.8, and Fig. 3 for more details).

Background estimates were obtained using a $4 \sigma$ clipped mean applied to pixel values in a region surrounding the source aperture, on a frame-by-frame basis. For channel 1, the whole sub-array frame was used, excluding a circular aperture of 6 pixel radius, centred on the source. For channels 2 and 4, an annulus of 1540 pixels was used, with the inner boundary set to avoid significant contributions from the wings of the point response function. Semistatic pixel masks ('pmasks'), provided along with the BCD frames, were applied to each frame and visible stars were also masked from the background estimates.

We estimated uncertainties in our measured flux values using a combination of the Poisson errors in the source counts and 
Table 1. Number of frames rejected as outliers based on flux and position measurements.

\begin{tabular}{lcccc}
\hline Channel & Flux & $x$-position & $y$-position & Total \\
\hline $1(3.6 \mu \mathrm{m})$ & 22 & 12 & 3 & $37(0.3$ per cent $)$ \\
$2(4.5 \mu \mathrm{m})$ & 24 & 0 & 0 & $24(1.5$ per cent $)$ \\
$4(8.0 \mu \mathrm{m})$ & 26 & 1 & 0 & $27(1.7$ per cent $)$ \\
\hline
\end{tabular}

the pixel-to-pixel variance in the background region. We use the variance in the background, rather than the average background count level, because the former is found to be significantly larger and is insensitive to constant offsets which could result from a poorly estimated zodiacal contribution. We also find that spatial gradients do not contribute significantly to our errors.

Along with flux measurements, image centroid estimates are essential for the removal of systematics affecting channels 1 and 2 . The ULTRACAM pipeline software has three options for determining the centroid of the source image: a cross-correlation of marginal sums in $x$ and $y$ with a 1D Gaussian of specified full width at half-maximum (FWHM; we used values of 1.6 pixels), a twodimensional Gaussian profile fit or a two-dimensional Moffat (1969) profile fit. We found that the cross-correlation technique gave consistently good residual rms values across the three channels when compared with the other centroid methods and so it was chosen for our final analysis. We note here that eclipse depths derived using the three different centroid techniques are all well within the $1 \sigma$ errors.

We rejected outliers in each channel based on flux measurements and centroid positions. Any frame with a flux, or $x$ or $y$ position more than $4 \sigma$ away from the median of the surrounding 150 (channel 1) or 20 (channels 2 and 4 ) frames was rejected. The total number of frames rejected in this procedure was 37 ( 0.3 per cent $)$ in channel 1 , 24 (1.5 per cent) in channel 2 and 27 (1.7 per cent) in channel 4. A break-down of these numbers is given in Table 1 .

In addition to this, we removed the first four data cubes for channel 1. These frames occurred when there was a drift in the telescope's pointing, and their fluxes do not correlate with position in the same way as the rest of the data. When these data are included, our derived eclipse depth for channel 1 changes by much less than $1 \sigma$, but with an increased rms on the normalized residuals. We also removed the first $10 \mathrm{~min}$ of data from channel 4 , based on our analysis in Section 3.6.

Binned versions of the light curves extracted from the aperture photometry are shown in the top panel of Fig. 4.

\subsection{Spitzer systematics}

\subsubsection{Channels 1 and 2}

Each of our secondary eclipse data sets displays systematic effects that have been well documented in the literature. Both the warm channel 1 and cryogenic channel 2 data are affected by a periodic variation in flux that correlates with the image centroid position (Charbonneau et al. 2005). This has been interpreted as the result of variations in sensitivity across individual pixels combined with a wobble in the pointing of the telescope (caused by the cycling of a heater on board Spitzer $)^{3}$. To model this effect, we tested sub-sets of the function

$\mathrm{d} f=a_{0}+a_{x} \mathrm{~d} x+a_{y} \mathrm{~d} y+a_{x y} \mathrm{~d} x \mathrm{~d} y+a_{x x} \mathrm{~d} x^{2}+a_{y y} \mathrm{~d} y^{2}+a_{t} \mathrm{~d} t$, where $\mathrm{d} f=f-\hat{f}$ is the stellar flux relative to its weighted mean, and $\mathrm{d} x=x-\hat{x}$ and $\mathrm{d} y=y-\hat{y}$ are the positions of the point spread function centre relative to their weighted means. We used unsmoothed positions to allow sampling of the short-time-scale jitter seen in Fig. 1. $\mathrm{d} t$ is the time since the first observation.

We also tested functions using a logarithmic time term, $a_{t} \ln (\mathrm{d} t+$ $t_{\text {off }}$ ), in place of the linear time term. The parameter $t_{\text {off }}$ was used to ensure that the argument of the logarithm did not go to 0 .

We select between these models in Section 3.6. For our final analysis, the channel 1 data were detrended using the function

$\mathrm{d} f=a_{0}+a_{x} \mathrm{~d} x+a_{y} \mathrm{~d} y+a_{x x} \mathrm{~d} x^{2}+a_{y y} \mathrm{~d} y^{2}$,

and for channel 2 we used

$\mathrm{d} f=a_{0}+a_{x} \mathrm{~d} x+a_{y} \mathrm{~d} y+a_{x x} \mathrm{~d} x^{2}+a_{y y} \mathrm{~d} y^{2}+a_{t} \mathrm{~d} t$.

\subsubsection{Channel 4}

The channel 4 data are affected by another well-known systematic, where the sensitivity of each pixel varies in time, dependent on its recent illumination history (Knutson et al. 2007). This gives rise to the so-called ramp clearly seen in the raw light curve (upper-right plot of Fig. 4). We tried detrending our channel 4 data using the following functions:

$\mathrm{d} f=a_{0}+a_{t} \mathrm{~d} t+a_{t t} \mathrm{~d} t^{2}$,

$\mathrm{d} f=a_{0}+a_{t} \ln \left(\mathrm{d} t+t_{\text {off }}\right)+a_{t t} \ln \left(\mathrm{d} t+t_{\text {off }}\right)^{2}$,

$\mathrm{d} f=a_{0}+a_{1} \exp \left(a_{2} \mathrm{~d} t\right)+a_{3} \exp \left(a_{4} \mathrm{~d} t\right)$,

where $\mathrm{d} f, \mathrm{~d} t$ and $t_{\text {off }}$ are as described previously. We also tried each of these functions without their final term [i.e. $a_{t t}=0$ for equations (4) and (5), and $a_{3}=0$ for equation (6)]. Agol et al. (2010) argue that equation (6) is a more physically motivated model for channel 4 data sets, under the assumption that the ramps are caused by charge trapping.

Again, we select between these models in Section 3.6, where the single exponential function is chosen for our final analysis:

$\mathrm{d} f=a_{0}+a_{1} \exp \left(a_{2} \mathrm{~d} t\right)$.

\subsection{Markov chain Monte Carlo parameter fitting}

We determined secondary eclipse light-curve solutions using a modified version of the Markov chain Monte Carlo (MCMC) algorithm implemented in various forms by Collier Cameron et al. (2007), Pollacco et al. (2008) and Anderson et al. (2011). From a set of proposal parameters $\left(T_{\mathrm{c}}, P,\left(R_{\mathrm{p}} / R_{\star}\right)^{2}, T_{14}, b, K_{1}, T_{\text {eff }},[\mathrm{Fe} / \mathrm{H}], \sqrt{e} \cos \omega\right.$, $\sqrt{e} \sin \omega, \Delta F_{3.6 \mu \mathrm{m}}, \Delta F_{4.5 \mu \mathrm{m}}$ and $\Delta F_{8.0 \mu \mathrm{m}}-$ see Table 2 for definitions), synthetic secondary eclipse light curves were computed as described in Wheatley et al. (2010) and fitted to our Spitzer data.

At each step in the Markov chain, a new set of proposal parameters is used to model the visible fraction of the planet, $\eta$, as a function of phase. For each IRAC channel, this is scaled by a factor $\Delta F$ and the stellar flux is estimated as

$f(\phi)=\frac{f^{\prime}(\phi)}{1+\eta(\phi) \Delta F}$,

where $f^{\prime}$ is the raw flux derived from the aperture photometry. From here the coefficients in equations (1)-(7) are obtained using singular value decomposition (Press et al. 1992). The terms $t_{\text {off }}, a_{2}$ and $a_{4}$ cannot be evaluated using this method and so they are introduced 

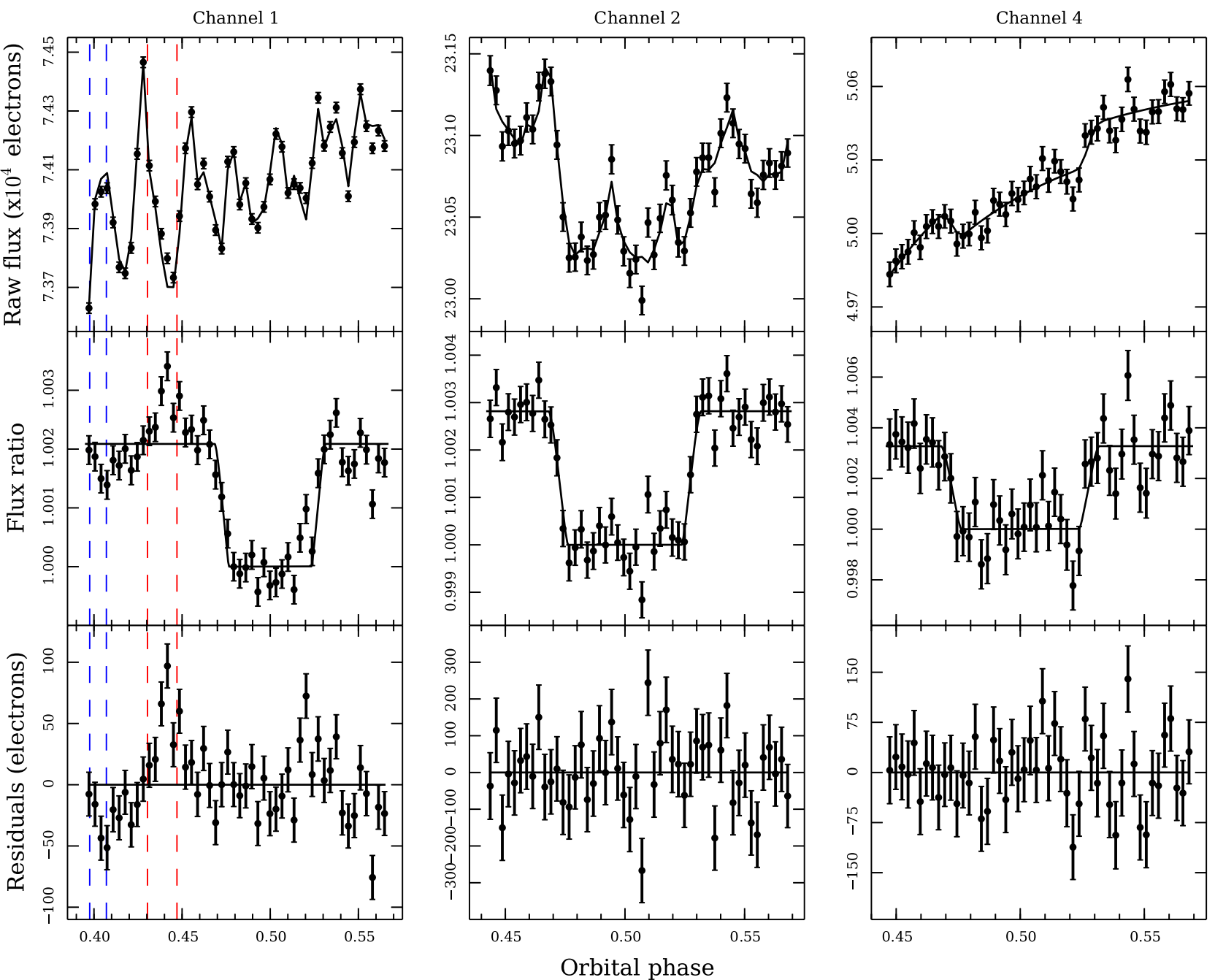

Figure 4. Columns from left to right are for channels 1,2 and 4, respectively. The top row shows the (binned) raw flux light curves for the optimal source aperture radii along with the full secondary eclipse model, which includes the detrending function. The middle row shows the flux with the detrending function removed and normalized to the flux of the star. Residuals are shown in the bottom row. The systematic effects described in the text are clearly seen in the raw data. These effects are generally well corrected for using the detrending functions given in equations (2), (3) and (7); however, there appear to be remaining features in the channel 1 data, which are discussed in the text. The eclipse depths, as displayed in the middle row, are $0.209,0.282$ and 0.328 percent in the three channels respectively. The blue and red dashed vertical lines for the channel 1 data correspond to the phase ranges of the positions highlighted in the same colours in the centroid position plot (Fig. 6). See Section 3.8 for a discussion of this.

as proposal parameters when they are in use. $t_{\text {off }}$ is also subject to a Gaussian prior to prevent its value from drifting to more than an hour or so.

For our final analysis, we scaled the error bars on our Spitzer photometry so that the reduced $\chi^{2}$ in each channel was 1 . This was done to ensure that realistic uncertainties on the proposal parameters were obtained from the MCMC. We did not perform this scaling when comparing the quality of fits provided by different detrending functions (see Section 3.6).

In the usual implementation of this MCMC code, transit data are also fitted to provide constraints on system parameters. In our implementation, we used Gaussian priors based on the light-curve model parameters from Southworth (2011), the transit ephemeris of Maciejewski et al. (2010), and stellar $T_{\text {eff }}$ and $[\mathrm{Fe} / \mathrm{H}]$ values from Pollacco et al. (2008). The motivation behind this was to take advantage of the numerous follow-up studies that have been made of WASP-3 in order to constrain, for example, the shape of the secondary eclipse, but to allow the dependences of the eclipse depth and timing on other parameters to be explored. In practice, we found no significant correlations between our eclipse constraints and other parameters (both proposal and priors).

We note that more recent ephemerides have been published (e.g. Sada et al. 2012; Nascimbeni et al. 2013). However, the uncertainties on our mid-eclipse phases are dominated by our data and the differences between ephemerides have a negligible effect (within $0.1 \sigma)$.

We fitted to the out-of-transit radial velocity data from Simpson et al. (2010) and Tripathi et al. (2010), since this puts constraints on the eccentricity and argument of periastron $(\omega)$ of the system. In conjunction, the timings of the secondary eclipses also constrain these parameters through the relation (Charbonneau et al. 2005)

$\Delta \phi \equiv \phi_{\mathrm{E}}-0.5 \simeq \frac{2}{\pi} e \cos \omega$ 
Table 2. WASP-3 system parameters derived from MCMC fitting to Spitzer secondary eclipse data (channels 1,2 and 4), using priors to constrain many properties of the system. Epoch and period priors are from Maciejewski et al. (2010), $T_{\text {eff }}$ and $[\mathrm{Fe} / \mathrm{H}]$ are from Pollacco et al. (2008), and others are from Southworth (2011). Parameter values in the column 'Channels 1, 2 and 4' are from the simultaneous fit to all the Spitzer data, whereas those in the column 'Channels 2 and 4' are from the fit to these two channels (see Section 3.9).

\begin{tabular}{|c|c|c|c|c|c|}
\hline Parameter & Symbol & Prior & Channels 1, 2 and 4 & Channels 2 and $4^{a}$ & Unit \\
\hline \multicolumn{6}{|l|}{ Fitted parameters: } \\
\hline Mid-transit time ${ }^{b}$ & $T_{\mathrm{c}}$ & $2454605.56000 \pm 0.00011$ & $2454640.64993 \pm 0.00011$ & $2454640.64993 \pm 0.00011$ & $\mathrm{~d}$ \\
\hline Orbital period & $P$ & $1.8468355 \pm 0.0000007$ & $1.8468355 \pm 0.0000007$ & $1.8468355 \pm 0.0000007$ & $\mathrm{~d}$ \\
\hline Stellar RV amplitude & $K_{1}$ & & $0.284 \pm 0.008$ & $0.284 \pm 0.008$ & $\mathrm{~km} \mathrm{~s}^{-1}$ \\
\hline Stellar temperature & $T_{\text {eff }}$ & $6400 \pm 100$ & $6400 \pm 100$ & $6400 \pm 100$ & $\mathrm{~K}$ \\
\hline Stellar metallicity & {$[\mathrm{Fe} / \mathrm{H}]$} & $0.0 \pm 0.2$ & $0.0 \pm 0.2$ & $0.0 \pm 0.2$ & \\
\hline Planet/star area ratio & $\left(R_{\mathrm{p}} / R_{\star}\right)^{2}$ & & $0.0112 \pm 0.0005$ & $0.0111 \pm 0.0005$ & \\
\hline Primary transit duration & $T_{14}$ & & $0.1143_{-0.0016}^{+0.0013}$ & $0.1155_{-0.0019}^{+0.0016}$ & $\mathrm{~d}$ \\
\hline Impact parameter & $b$ & & $0.527 \pm 0.019$ & $0.511 \pm 0.020$ & \\
\hline$c$ & $\begin{array}{l}\sqrt{e} \cos \omega \\
\sqrt{e} \sin \omega\end{array}$ & & $\begin{array}{c}-0.009_{-0.012}^{+0.025} \\
0.019_{-0.061}^{+0.076}\end{array}$ & $\begin{array}{c}-0.010_{-0.013}^{+0.017} \\
0.021_{-0.065}^{+0.083}\end{array}$ & \\
\hline Channel $1(3.6 \mu \mathrm{m})$ eclipse depth ${ }^{c}$ & $\Delta F_{3.6 \mu \mathrm{m}}$ & & $0.209_{-0.028}^{+0.040}$ & & per cent \\
\hline Channel $2(4.5 \mu \mathrm{m})$ eclipse depth & $\Delta F_{4.5} \mu \mathrm{m}$ & & $0.282 \pm 0.012$ & $0.282 \pm 0.012$ & per cent \\
\hline Channel $4(8.0 \mu \mathrm{m})$ eclipse depth ${ }^{c}$ & $\Delta F_{8.0 \mu \mathrm{m}}$ & & $0.323_{-0.054}^{+0.081}$ & $0.328_{-0.055}^{+0.086}$ & per cent \\
\hline \multicolumn{6}{|l|}{ Derived parameters: } \\
\hline Orbital separation & $a$ & & $0.0315 \pm 0.0003$ & $0.0315 \pm 0.0003$ & au \\
\hline Orbital inclination & $i$ & $83.72 \pm 0.39$ & $83.91 \pm 0.28$ & $84.06 \pm 0.29$ & $\circ$ \\
\hline$c$ & $e \cos \omega$ & & $-0.0005_{-0.0005}^{+0.0017}$ & $-0.0006_{-0.0006}^{+0.0010}$ & \\
\hline \multirow{3}{*}{ Orbital eccentricity } & $e \sin \omega$ & & $0.001_{-0.002}^{+0.011}$ & $0.001_{-0.003}^{+0.014}$ & \\
\hline & $e$ & & $0.003_{-0.002}^{+0.011}$ & $0.003_{-0.002}^{+0.013}$ & \\
\hline & & & $<0.045(3 \sigma)$ & $<0.049(3 \sigma)$ & \\
\hline Mid-eclipse phase ${ }^{c}$ & $\phi_{\mathrm{E}}$ & & $0.4997_{-0.0003}^{+0.0011}$ & $0.4996_{-0.0004}^{+0.0007}$ & \\
\hline Mid-eclipse time $(\mathrm{ch} 1)^{b, c, d}$ & $T_{\mathrm{E}, 1}$ & & $2455130.98459_{-0.00065}^{+0.00200}$ & & $\mathrm{~d}$ \\
\hline Mid-eclipse time $(\operatorname{ch} 2 \text { and } 4)^{b, c, d}$ & $T_{\mathrm{E}, 2 / 4}$ & & $2454728.37446_{-0.00065}^{+0.00193}$ & $2454728.37432_{-0.00078}^{+0.00108}$ & $\mathrm{~d}$ \\
\hline Secondary eclipse duration & $T_{58}$ & & $0.1147 \pm 0.0011$ & $0.1161 \pm 0.0013$ & $\mathrm{~d}$ \\
\hline Stellar mass & $M_{\star}$ & & $1.23 \pm 0.03$ & $1.23 \pm 0.03$ & $\mathrm{M}_{\odot}$ \\
\hline Stellar radius & $R_{\star}$ & & $1.36 \pm 0.02$ & $1.36 \pm 0.02$ & $\mathrm{R} \odot$ \\
\hline Scaled stellar radius & $R_{\star} / a$ & $0.1994 \pm 0.0032$ & $0.2004 \pm 0.0025$ & $0.2011 \pm 0.0026$ & \\
\hline Stellar density & $\rho_{\star}$ & & $0.488_{-0.017}^{+0.018}$ & $0.482_{-0.018}^{+0.019}$ & $\rho \odot$ \\
\hline Stellar surface gravity & $\log g_{\star}$ & & $4.260 \pm 0.011$ & $4.256 \pm 0.011$ & $(\mathrm{cgs})$ \\
\hline Planet mass & $M_{\mathrm{p}}$ & & $1.98 \pm 0.07$ & $1.98 \pm 0.06$ & $\mathrm{M}_{\mathrm{J}}$ \\
\hline Planet radius & $R_{\mathrm{p}}$ & & $1.40 \pm 0.03$ & $1.40 \pm 0.03$ & $\mathrm{R}_{\mathrm{J}}$ \\
\hline Scaled planetary radius & $R_{\mathrm{p}} / a$ & $0.02125 \pm 0.00041$ & $0.02122 \pm 0.00042$ & $0.02120 \pm 0.00042$ & \\
\hline Planet density & $\rho_{\mathrm{p}}$ & & $0.72 \pm 0.05$ & $0.72 \pm 0.05$ & $\rho_{J}$ \\
\hline Planet surface gravity & $\log g_{\mathrm{p}}$ & & $3.36 \pm 0.02$ & $3.36 \pm 0.02$ & $(\mathrm{cgs})$ \\
\hline Channel 1 brightness temperature & $T_{3.6 \mu \mathrm{m}}$ & & $2280_{-150}^{+210}$ & & $\mathrm{~K}$ \\
\hline Channel 2 brightness temperature & $T_{4.5 \mu \mathrm{m}}$ & & $2390 \pm 80$ & $2400 \pm 80$ & $\mathrm{~K}$ \\
\hline Channel 4 brightness temperature & $T_{8.0 \mu \mathrm{m}}$ & & $2190_{-260}^{+370}$ & $2210_{-250}^{+390}$ & $\mathrm{~K}$ \\
\hline
\end{tabular}

${ }^{a}$ Final values are taken from this column, except for the eclipse depth, brightness temperature and mid-eclipse time for channel 1.

${ }^{b} \mathrm{HJD}(\mathrm{TT})$.

${ }^{c}$ For these parameters, the prayer bead analysis led to an increase in the error estimates.

${ }^{d}$ These are the observed mid-eclipse times, i.e. they do not account for the light travel time (of $31.4 \mathrm{~s}$ ) across the system. Note that the mid-eclipse times in the first column are derived from the joint fit to both eclipse events, not from individual fits to each eclipse.

where $\phi_{\mathrm{E}}$ is the observed mid-eclipse phase and 0.5 is the expectation from a circular orbit.

\subsection{Light travel time corrections}

We accounted for the light travel time across the WASP-3 system for each of our data sets. For a circular orbit, this will cause the secondary eclipse to appear later than the $\phi=0.5$ prediction by $\frac{2 a}{c P}$. Here $a$ is the radius of the orbit and $c$ is speed of light. This delay occurs because the epoch of the orbit is given for mid-transit. Similarly, $\phi=0$ for the radial velocity measurements will occur $\frac{a}{c P}$ later than mid-transit. We subtracted $31.4 \mathrm{~s}$ from the secondary eclipse timings and $15.7 \mathrm{~s}$ from the radial velocity timings to account for this. These values were estimated using a value for $a$ from an initial MCMC run (without these corrections) and assuming a circular orbit. We found that our value for $a$ is not affected by the correction, and that the light travel time correction did not change significantly for eccentric orbits allowed by our final results. 
We did not correct for the fact that some times were given as $\mathrm{He}-$ liocentric Julian Dates (HJD), while others were given as Barycentric Julian Dates (BJD). The differences here are typically $\sim 1 \mathrm{~s}$, much smaller than our final errors on the central eclipse time, and so will not have significantly impacted our results.

\subsection{Detrending model selection}

We assessed the relative quality of the fits given by the functions described in Sections 3.3.1 and 3.3.2 using the Bayesian information criterion (BIC; Schwarz 1978):

$\mathrm{BIC}=\chi^{2}+k \ln N$,

where $k$ is the number of free model parameters and $N$ is the number of data points used. This is used to ensure that simpler models are favoured.

We found that equations (2) and (3) were the most appropriate detrending functions according to the BIC, for channels 1 and 2, respectively. Other functions that gave similar BIC values resulted in eclipse depths well within $1 \sigma$ of our final result.

For channel 4, we found that the single exponential function (equation 7) was marginally favoured by the BIC, with the linear logarithmic time [equation (5) with $a_{t t}=0$ ] and double exponential (equation 6) functions giving similarly good fits. We found differences in the resulting eclipse depths of $\sim 1 \sigma$, which stemmed from the data at the beginning of the time series where the single exponential function did not give a good fit. With the first $10 \mathrm{~min}$ of data removed, these three models gave consistent channel 4 eclipse depths, with the single exponential again being favoured by the BIC. As a result, we detrended our channel 4 light curve using the single exponential function with the first $10 \mathrm{~min}$ of data removed.

\subsection{Source aperture size}

We derived MCMC solutions for light curves extracted using source aperture radii between 2.0 and 6.0 pixels. The optimal radii were found by assessing where the rms of the normalized residuals from the fitted light curve was minimized. Values of 2.5, 3.0 and 2.5 pixels were found for channels 1, 2 and 4, respectively. Fig. 3 highlights these choices. We plot the signal-to-noise ratios of flux measurements from a typical image, the rms of the normalized residuals and the eclipse depths as a function of source aperture radius for our three channels. Minima in the rms values coincide well with maxima in the typical signal-to-noise values, as expected. In all channels, the eclipse depths remain well within the MCMC uncertainties, except for the larger radii in channel 1, which differ from those at smaller radii at the $2 \sigma$ level. However, there is a significant increase in the rms of the normalized residuals, along with an associated drop in typical signal-to-noise, at larger radii due to the inclusion of significantly more background photons.

For channels 1 and 4, Fig. 3 shows a discrepancy between the estimated and actual rms values at our chosen radii. The trends in these discrepancies with radius suggest that we are hitting a noise floor at smaller radii, where some additional source of noise (not accounted for in our flux error estimation) becomes significant.

Fig. 4 shows binned time series for the three IRAC channels, using our chosen source apertures. The top row shows the raw flux light curves with the full secondary eclipse model, including the detrending function. The middle row shows the flux with the detrending function removed and normalized to the flux of the star, along with the eclipse model. The model residuals are shown in the bottom row.

\subsection{Channel 1 systematics}

Fig. 4 shows that for channel 1 , systematic features remain in the residuals. Given the variation of eclipse depth with source aperture radius in this channel (see Fig. 3), we considered the possibility that the systematic noise could be reduced at larger radii. In Fig. 5, we show normalized residual rms values as a function of bin width for different radii in each of the three channels used. In channel 1, red noise is clearly seen as the rms values increase with respect to the white noise expectation for large bin sizes. Crucially, this feature is present at large radii ( $r=5.0$ pixels) as well as at our chosen
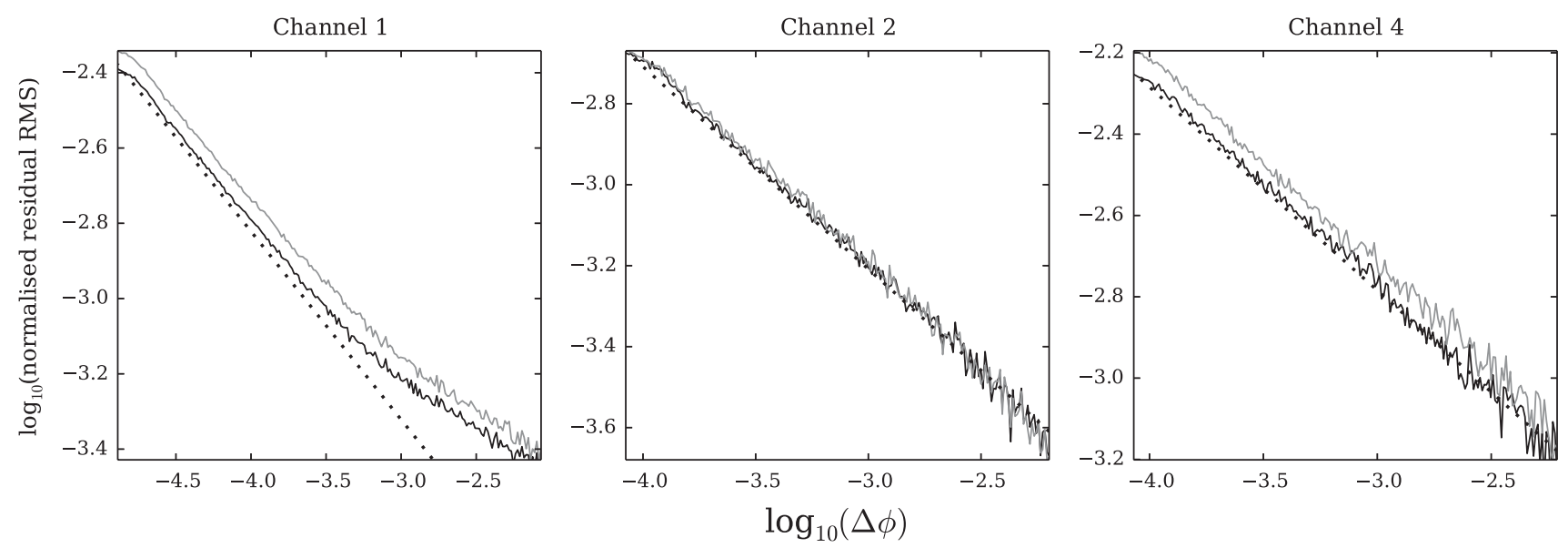

Figure 5. Binned normalized residual rms values as a function of bin width. From left to right, plots are for channels 1,2 and 4 . Black lines are for light curves extracted using the optimal source aperture radii $(2.5,3.0$ and 2.5 pixels, respectively) and the grey lines are for a source aperture of 5 pixels. Dotted lines show the $1 / \sqrt{n}$ expectation, where $n$ is the number of data points per bin. This expectation is fixed to the unbinned rms value for the optimal source aperture radius. Systematic noise is clearly present in the channel 1 data across the range of aperture sizes and does not decrease significantly at large radii. Since the signal-to-noise and normalized residual rms values degrade at these larger radii, there is no motivation in using a larger aperture. Channels 2 and 4 show well-behaved trends, following the $1 / \sqrt{n}$ expectation. The offsets in rms values between the different radii reflect the increases in white noise seen in the top two panels of Fig. 3. 


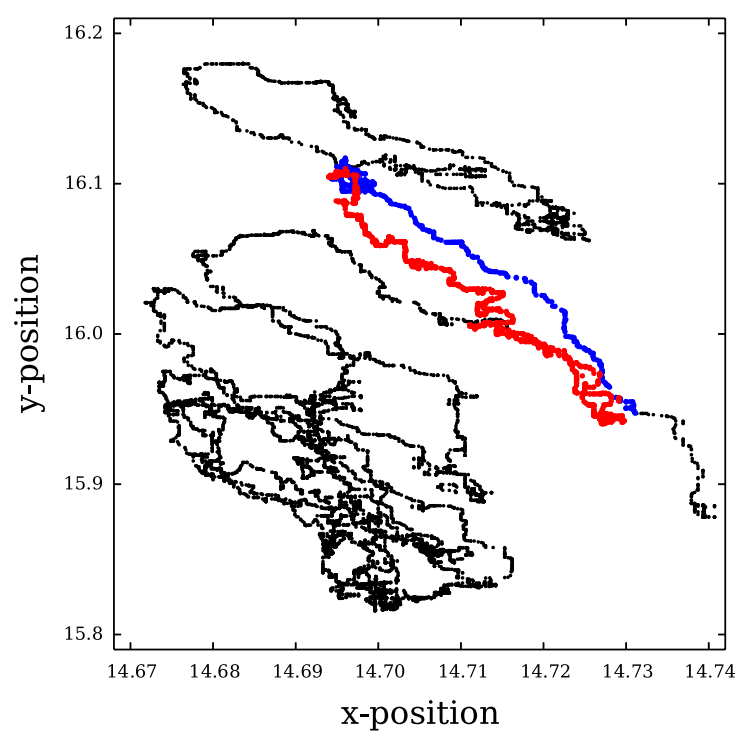

Figure 6. Median-filtered centroid positions for the channel 1 data set (with a filter width of 200 frames). At the start of the observations, the centroid position is $x \sim 14.74, y \sim 15.9$. Note the significantly larger scale in $y$ compared to $x$. Throughout the first $\sim 4 \mathrm{~h}$ of observations, the image moves across a large range in $y$, before settling down for the final $\sim 3 \mathrm{~h}$. The blue and red highlighted regions correspond to those marked in Fig. 4, and are discussed in the text.

radius of 2.5 pixels. Thus, there is no motivation for using the larger aperture.

The origin of the systematic noise in the channel 1 residuals is not clear. We looked for clues in terms of the centroid position and FWHM of WASP-3, but do not find any strong correlations with the residuals. There is a large movement of the image centroid in $y$ from the beginning of the observations, covering a distance of $\sim 0.6$ pixels in $\sim 4 \mathrm{~h}$ (Fig. 6). This is where the most clear systematic is also present, so we suspect that this motion is playing a significant role. However, as shown in Figs 4 and 6, the extremes of a slope in the residuals ( $\phi=0.40-0.44)$ occur while the centroid positions cover very similar parts of the pixel, demonstrating why a simple detrending function in space and time cannot correct these features.

We note that Kepler light curves of F-type stars with similar spin periods to WASP-3 (Mathur et al. 2014) show photometric variability with amplitudes and time-scales comparable to our channel 1 systematic features. It is therefore plausible that they result from stellar variability rather than a detector-related systematic.

\subsection{Prayer bead errors}

To estimate the extent to which the systematic noise affects our eclipse depth and timing measurements, we performed a 'prayer bead' analysis (e.g. Gillon et al. 2007), where light-curve residuals are subtracted from the raw data and then added back in with a cyclic offset in phase. We used 20 different offsets, equally spaced across the phases sampled, and we applied these shifts to each of our light curves. The resulting data sets were run through our MCMC procedure as described previously. From the resulting distribution, we assessed the $1 \sigma$ errors as the 68 percent confidence interval centred on the value obtained from the original MCMC fit. The error values in Table 2 were taken as the larger of either this error or that given by our MCMC analysis on the unmodified data.

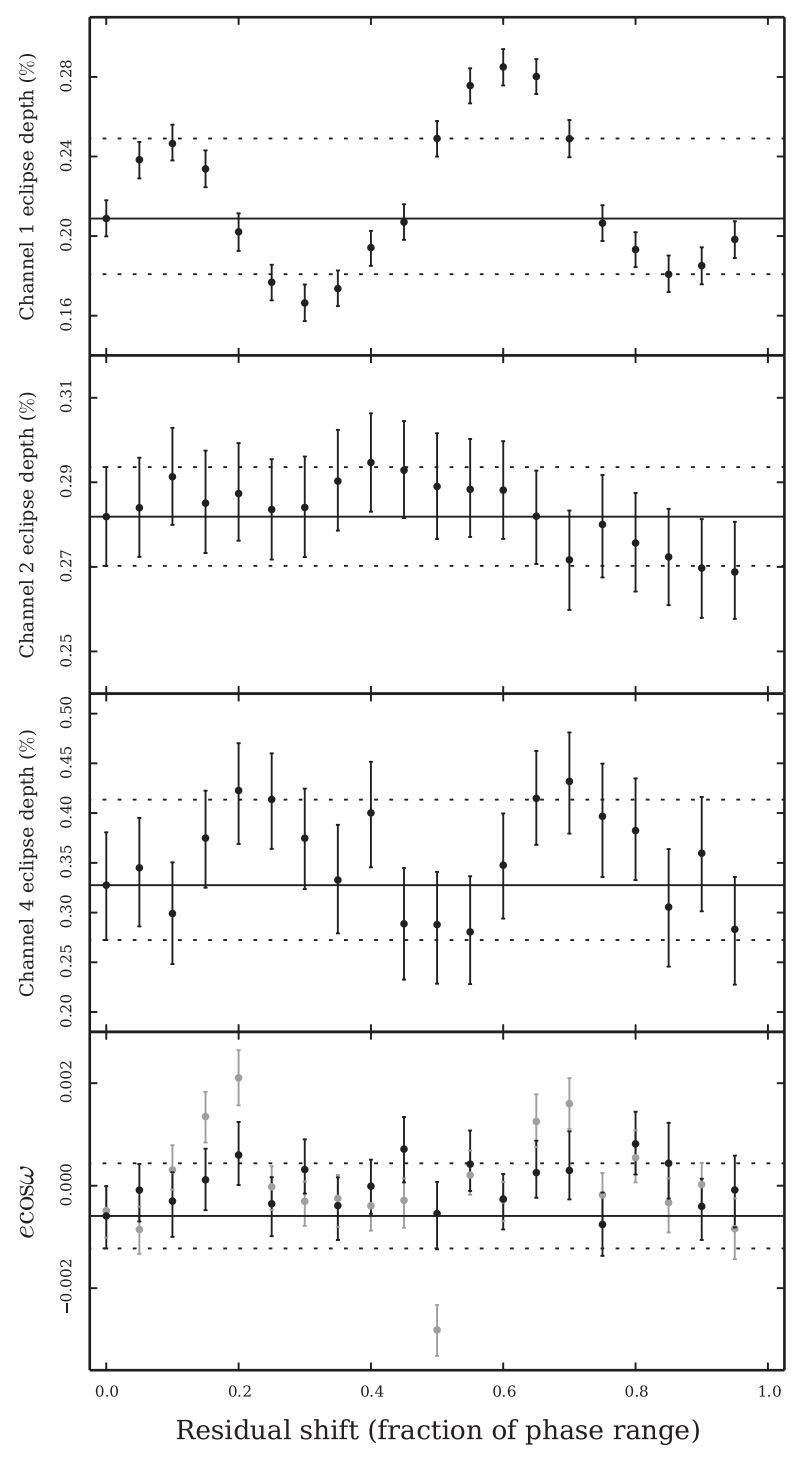

Figure 7. Eclipse depth values, with MCMC errors, for the fake data sets created by adding residuals back into our best-fitting model, with a given offset. The dotted horizontal lines give our final adopted errors. The top three panels are for the eclipse depths in channels 1,2 and 4, respectively, and the bottom panel is for $e \cos \omega$. Channel 1 is clearly the most affected by these shifts, as evidenced by the correlated noise signatures seen in Figs 4 and 5 . We adopt the prayer bead errors here since they are clearly more representative than the errors derived from the MCMC code. The values for the channels 2 and 4 eclipse depths were derived from the second prayer bead analysis (which excluded the channel 1 data). The residuals here are much cleaner and the variation in eclipse depths is comparable to the MCMC error bars. For $e \cos \omega$, we plot values from both prayer bead analyses (those from the second analysis are shown in black), showing an improvement in the distribution when the channel 1 data are excluded. However, the positive error bar is still significantly affected by the residual permutations.

The only parameters for which prayer bead errors were larger were the channel 1 and 4 eclipse depths and the parameters associated with the eclipse timings $\left(\sqrt{e} \cos \omega, e \cos \omega, \phi_{\mathrm{E}}, T_{\mathrm{E}, 1}, T_{\mathrm{E}, 2 / 4}\right)$. Fig. 7 shows the results of the prayer bead MCMC runs for the eclipse depths and for $e \cos \omega$. As expected, the channel 1 eclipse depth is the most affected, with its errors increasing by a factor of 3-4 above the MCMC errors. 
Since the eclipse timing $(e \cos \omega)$ is constrained by each channel simultaneously, we felt it appropriate to re-run our prayer bead analysis with the channel 1 data removed. This was done because systematics associated with that channel could have an adverse effect on our determinations of $e \cos \omega$, as well as the channel 2 and 4 eclipse depths. The resulting distribution for $e \cos \omega$ does show an improvement, though the positive error is still larger than the MCMC error. There is little change to the channel 2 and 4 eclipse depth distributions after dropping the channel 1 data. The channel 2 prayer bead variation is smaller than the MCMC errors, and for channel 4 only the positive error is set by the prayer bead error.

\section{RESULTS}

The results from our MCMC analyses of the Spitzer secondary eclipse data can be found in Table 2. They are given for our optimal source aperture radii, with errors accounting for the results of the prayer bead analysis. The first column of results includes secondary eclipse data from channels 1, 2 and 4, while the second column does not include the channel 1 data (see Section 3.9). We adopt the parameters in the second column as our final set. The exceptions to this are the eclipse depth, brightness temperature and mid-eclipse time for channel 1, which are taken from the first column.

We find eclipse depths of $0.209_{-0.028}^{+0.040}, 0.282 \pm 0.012$ and $0.328_{-0.055}^{+0.086}$ percent in channels 1,2 and 4 , respectively. These eclipse depths are equivalent to planet-to-star flux ratios. Using simple blackbody estimates, we expect the planetary thermal emission to dominate the planetary reflected light across these wavelengths. For example, using a very conservative value of 0.5 for the Bond albedo, the ratio of thermally emitted photons to reflected photons from the planet is $20-40$ across the IRAC channels. We therefore interpret our secondary eclipse measurements as resulting from the thermal emission of the planet.

We used the measured eclipse depths to estimate brightness temperatures for each channel. These were found by integrating the photon flux of a blackbody over the response function for the channel and dividing this by the stellar flux (using the models of Kurucz 1979) integrated over the same response function (Harrington et al. 2007). The brightness temperature was iterated until this quantity was matched to the relevant eclipse depth. We find values of $T_{3.6 \mu \mathrm{m}}=2280_{-150}^{+210} \mathrm{~K}, T_{4.5 \mu \mathrm{m}}=2400 \pm 80 \mathrm{~K}$ and $T_{8.0 \mu \mathrm{m}}=2210_{-250}^{+390} \mathrm{~K}$.

Our final result for $e \cos \omega$ is $-0.0006_{-0.0006}^{+0.0010}$, with the positive error resulting from the prayer bead analysis and the negative error from the MCMC. This result suggests that the timing of the eclipse is not significantly different from the expectation for a circular orbit. This implies that the eccentricity of the system can only be large if $\omega \simeq 90^{\circ}$. For example, for $e>0.03, \omega$ is restricted to $87.5<|\omega|<$ 94.2 at $3 \sigma$. A measurement of $e \cos \omega=0.0070 \pm 0.0032$ by Zhao et al. (2012) gives a marginal $2 \sigma$ agreement with our result.

Since the prayer bead analysis does not affect our eccentricity estimates, we use the MCMC distribution for our constraints, which gives a result of $e=0.003_{-0.002}^{+0.013}$. Our $3 \sigma$ upper limit on the eccentricity is 0.049 .

\section{DISCUSSION}

\subsection{Albedo and energy redistribution}

Two key properties in our understanding of exoplanetary atmospheres are the fraction of the incident stellar radiation that is reflected by the planet (the Bond albedo) and the efficiency with which the planet redistributes the absorbed energy around the planet.

These properties strongly influence the planetary day-side flux and hence the expected secondary eclipse depths. While thermal emission measurements alone cannot break the degeneracy that exists between these two properties (an increase in the Bond albedo is indistinguishable from an increase in the efficiency of heat redistribution), they can still provide useful constraints.

To do this, we follow the methodology of Cowan \& Agol (2011). First, we estimate the day-side effective temperature $\left(T_{\mathrm{d}}\right)$ of WASP-3b using

$\int_{0}^{\infty} B_{\lambda}(T(\lambda)) \mathrm{d} \lambda=\frac{\sigma}{\pi} T_{\mathrm{d}}^{4}$,

where $T(\lambda)$ is given by a piecewise model of the brightness temperatures derived in Section 4. The model is linear between the wavelength bands and constant longwards (shortwards) of the longest (shortest) wavelength band.

Using equation (11), we find a day-side effective temperature of $T_{\mathrm{d}}=2280_{-150}^{+200} \mathrm{~K}$, where the error was assessed using a Monte Carlo technique.

Constraints on the Bond albedo and heat redistribution can be made through a simple parametrization of the day-side effective temperature, again from Cowan \& Agol (2011):

$T_{\mathrm{d}}=T_{0}\left(1-A_{\mathrm{B}}\right)^{1 / 4}\left(\frac{2}{3}-\frac{5}{12} \varepsilon\right)^{1 / 4}$.

Here $A_{\mathrm{B}}$ is the Bond albedo and $T_{0}$ is the temperature of the substellar point (assuming $A_{\mathrm{B}}=0$ ). Heat redistribution is described in the final term of this equation, where $\varepsilon$ can vary between 0 (instantaneous re-radiation) and 1 (fully redistributed heat, i.e. the planet radiates isotropically).

Using the system parameters in Table 2, we find $T_{0}=2870 \pm$ $50 \mathrm{~K}$, giving $T_{\mathrm{d}} / T_{0}=0.79_{-0.06}^{+0.07}$. Our error here includes a component that accounts for the systematic error expected from the linear interpolation method of Cowan \& Agol (2011). Fig. 8 shows the resulting marginalized $1 \mathrm{D}$ probability distributions of $\varepsilon$ and $\mathrm{A}_{\mathrm{B}}$. The $1 \sigma$ confidence limits are 0.53 and 0.34 for these two parameters, respectively. The $3 \sigma$ limits are 0.99 and 0.72 .

Cowan \& Agol (2011) have highlighted an emerging trend for highly irradiated planets (with high $T_{0}$ values) to have low albedo and energy redistribution efficiency values (i.e. high $T_{\mathrm{d}} / T_{0}$ values). With $T_{0}=2870 \pm 50 \mathrm{~K}$ and $T_{\mathrm{d}} / T_{0}=0.79_{-0.06}^{+0.07}$, WASP- $3 \mathrm{~b}$ is another case of such a planet. We note here that this result is based on our Spitzer data only. Including a $K_{\mathrm{s}}$ brightness temperature based on the results of Zhao et al. (2012) increases this value to $T_{\mathrm{d}} / T_{0}=$ $0.94 \pm 0.05$.

\subsection{Atmospheric modelling}

In Fig. 9, we compare our eclipse depths with the 1D plane-parallel models of Fortney et al. (2008). These models assume solar metallicity, with abundances from Lodders (2003), and are cloud-free. Atmospheric compositions are found under the assumption of chemical equilibrium throughout the atmosphere. Only two model inputs are varied. First, in order to simulate varying degrees of redistribution of incident stellar energy around the planet (via atmospheric winds), the irradiating flux at the top of the atmosphere is weighted by a geometric factor $(f)$. Models were produced for $f=1 / 4$ to simulate isotropic planetary radiation, $f=1 / 2$ for even emission over the day side only and $f=2 / 3$ to simulate instantaneous reradiation of flux by the planet (e.g. Burrows, Budaj \& Hubeny 

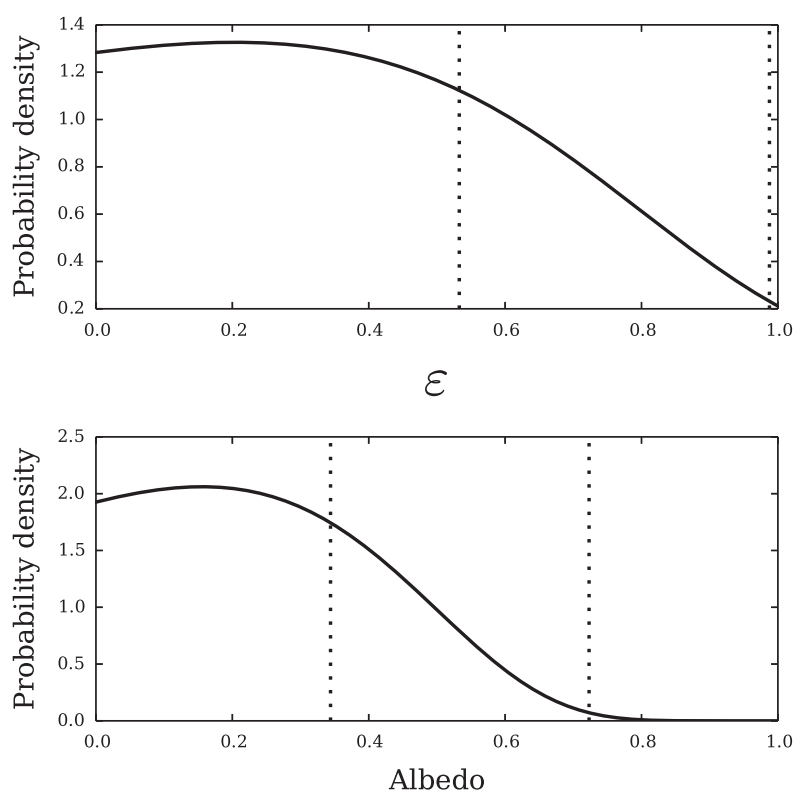

Figure 8. Marginalized 1D probability density functions for the heat redistribution parameter and the albedo for WASP-3b. A 2D probability distribution is created using our $T_{\mathrm{d}} / T_{0}$ value (derived using our measured Spitzer brightness temperatures) with equation 7 in Cowan \& Agol (2011). This is evaluated over a grid of $A_{\mathrm{B}}$ and $\varepsilon$ values. The marginal distributions for each parameter are found by integrating this $2 \mathrm{D}$ distribution over the allowable range of the other parameter. The dotted lines show the $1 \sigma$ and $3 \sigma$ confidence limits.
2008; Hansen 2008; note also that $f=1 / 4$ is equivalent to $\varepsilon=1$ and $f=2 / 3$ is equivalent to $\varepsilon=0$ in equation 12). Secondly, models are produced either with $\mathrm{TiO}$ and $\mathrm{VO}$ present in the atmosphere with their equilibrium abundances ('TiO' in Fig. 9), or with these molecules removed at $P<10$ bars ('no TiO'). This is motivated by the apparent inverted/non-inverted dichotomy amongst hot Jupiter exoplanets. For the 'TiO' case, regardless of our choice of $f$, the atmospheric temperatures of WASP-3 always lead to significant amounts of gaseous phase $\mathrm{TiO} / \mathrm{VO}$ in the upper atmosphere, which drives temperature inversions. Conversely, the 'no TiO' cases lack inversions. The differing temperature structures of these models are highlighted in Fig. 10, which also shows the relative contributions of different layers in the modelled atmospheres to the flux in each of our Spitzer bands.

For both 'TiO' and 'no TiO' models, we find that inefficient heat redistribution is heavily favoured (Fig. 9), in agreement with the conclusion from the effective temperature estimate. For the 'TiO' models, our data are best reproduced in the $f=1 / 2$ case, whereas for the 'no TiO' models the extreme $f=2 / 3$ case is favoured.

Our passband-integrated model predictions for the 'TiO' model provide good agreement to our measurements in all three Spitzer bands. The 'no TiO' model reproduces the channel 1 and 4 eclipse depths well; however, the channel 2 model prediction is discrepant, at $3 \sigma$. The fact that this is our most robust measurement, and that the 'no TiO' model uses the maximum reasonable value for $f$ (Hansen 2008), argues strongly in favour of the 'TiO', inverted atmosphere case.

We also compare these models to the $K_{\mathrm{s}}$-band measurement from Zhao et al. (2012) and find that neither reproduces the very high

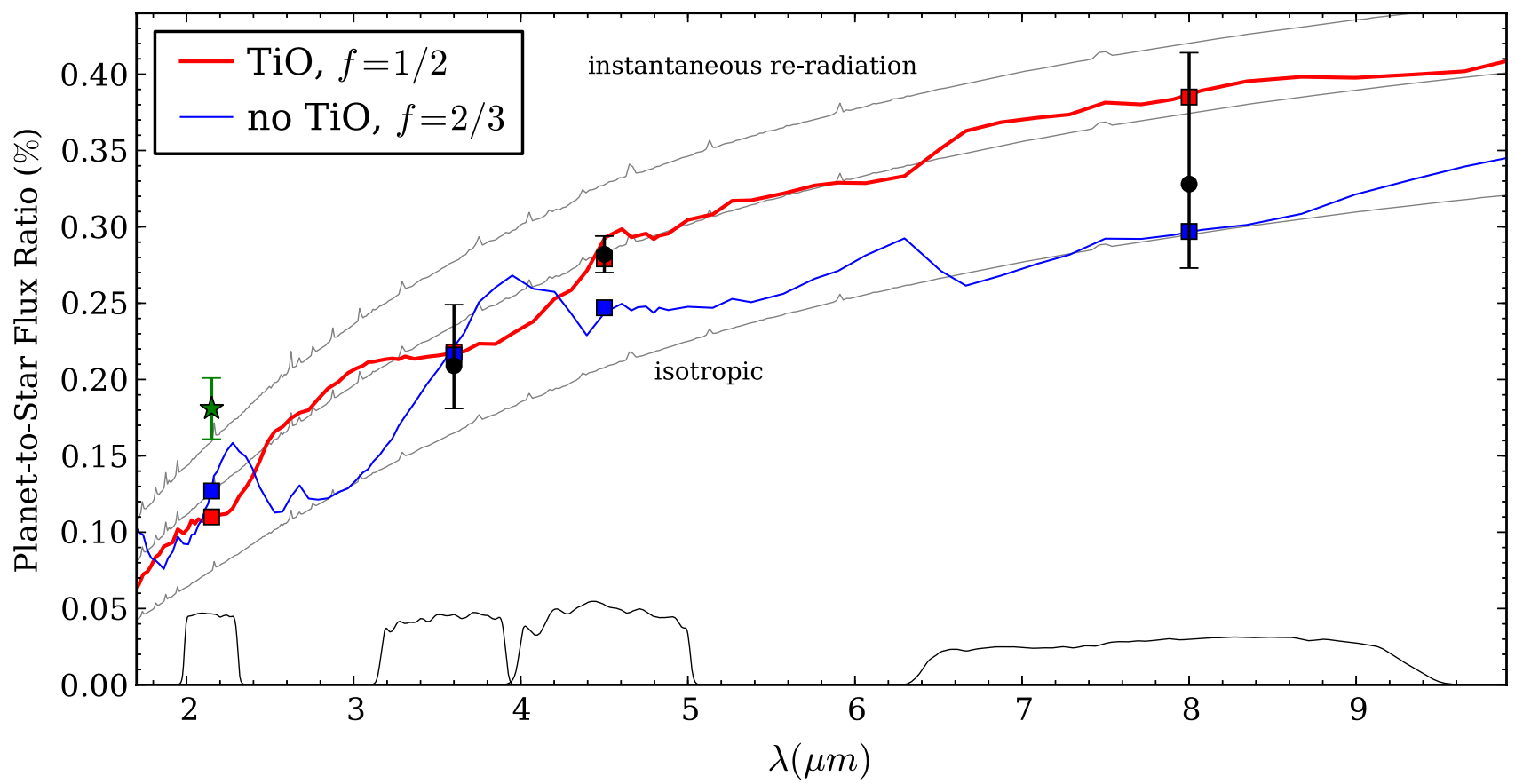

Figure 9. Spitzer/IRAC eclipse depths are shown along with the $K_{\mathrm{s}}$-band eclipse depth (green star) found by Zhao et al. (2012). The red curve is the Fortney et al. (2008) model for the case where $\mathrm{TiO}$ and $\mathrm{VO}$ are present in their equilibrium abundances. The blue curve is the same, but with TiO and VO removed at $P<10$ bars. The passband-integrated model predictions are given as squares in the same colours. Comparisons of these models to the data are given in the text. The grey curves use planetary blackbody models with Kurucz (1979) stellar models for WASP-3. The top and bottom curves give the extremes of the expected planetary day-side temperature, assuming a Bond albedo of 0 . The lower curve is for isotropic re-radiation $(\varepsilon=1$ in equation 12$)$ and the upper curve is for instantaneous re-radiation $(\varepsilon=0)$. For reference, the upper grey curve has a temperature of $2590 \mathrm{~K}$, and the lower has a temperature of $2030 \mathrm{~K}$. The middle grey curve is the best-fitting blackbody to the Spitzer bands, with a temperature of $2390 \mathrm{~K}$. IRAC response curves for channels 1,2 and 4 are also shown with arbitrary units, along with the $K_{\mathrm{s}}$-band response curve. 


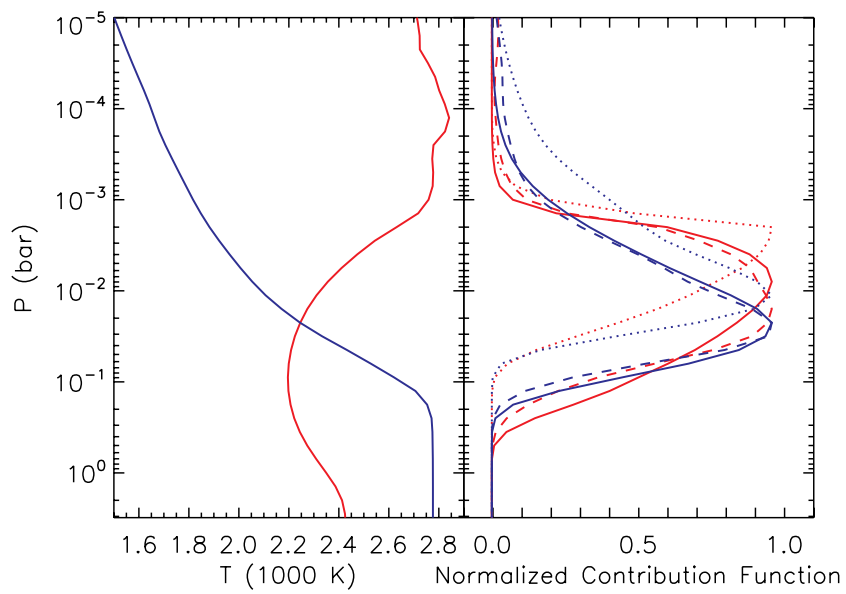

Figure 10. Left-hand panel: atmospheric pressure-temperature profiles for the models presented in Fig. 9 (using the same colour scheme). Significantly different temperature structures can be seen, with an inversion occurring for the red ('TiO', $f=1 / 2$ ) model at $P \sim 10^{-1}$ bar. Right-hand panel: normalized contribution functions for our three Spitzer/IRAC bandpasses. Solid lines are for channel $1(3.6 \mu \mathrm{m})$, dashed lines for channel $2(4.5 \mu \mathrm{m})$ and dotted lines are for channel $4(8.0 \mu \mathrm{m})$. At wavelengths where we see less deep into the atmosphere (i.e. $8 \mu \mathrm{m}$ ), the inverted atmosphere is brighter. This difference is what drives the difference in the models plotted in Fig. 9.

brightness temperature found. A similar situation has been found for the system HAT-P-1 (de Mooij et al. 2011), though with planetary brightness temperatures typically $\sim 700 \mathrm{~K}$ lower than WASP-3b. de Mooij et al. (2011) highlight that for HAT-P-1b making the lower atmosphere hotter would account for their $K_{\mathrm{s}}$ measurement, since $\mathrm{H}_{2} \mathrm{O}$ opacity windows across this band result in sampling flux from these deeper regions. However, for their models such a change would also increase the channel 1 eclipse depth prediction to be inconsistent with the measurement from Todorov et al. (2010). Comparing the two models in Fig. 9, the 'no TiO' case provides a better fit in the $K_{\mathrm{s}}$ band, due to its relatively hotter lower atmosphere, but it still underestimates the eclipse depth by $\sim 3 \sigma$. This suggests that the presence or absence of $\mathrm{TiO}$ and $\mathrm{VO}$ is not driving this discrepancy. As a result, we still favour our inverted atmosphere conclusion based on the Spitzer measurements.

The distinction in our data between the two vertical temperature structures is driven mainly by the $4.5 \mu \mathrm{m}$ eclipse depth. Its relatively high value compared to the $3.6 \mu \mathrm{m}$ measurement favours the presence of $\mathrm{H}_{2} \mathrm{O}$ and $\mathrm{CO}$ being seen in emission (a result of a temperature inversion). This fact has been used in the empirical measure suggested by Knutson et al. (2010). Following Anderson et al. (2011), we define this measure $(\zeta)$ as the gradient of the measurements at 3.6 and $4.5 \mu \mathrm{m}$, i.e. $\left(\Delta F_{4.5 \mu \mathrm{m}}-\Delta F_{3.6 \mu \mathrm{m}}\right) / 0.9 \mu \mathrm{m}$, minus the gradient of the blackbody that is the best fit to the two measurements. Planets giving smaller $\zeta$ values tend not to show inverted atmospheres, whereas those with inversions tend to have larger $\zeta$ values, with a cut-off at around $\zeta \sim-0.05$ per cent $\mu \mathrm{m}^{-1}$. For WASP-3b, we find $\zeta=0.027 \pm 0.046$ per cent $\mu \mathrm{m}^{-1}$, placing it in the group of planets with inverted atmospheres.

\subsection{Stellar activity correlation}

In Fig. 11, we reassess the possible correlation between $\zeta$ and the activity of the host star (quantified using the $\log R_{\mathrm{HK}}^{\prime}$ index), as suggested by Knutson et al. (2010). WASP-3 lies near the proposed activity cut-off between stars hosting planets with

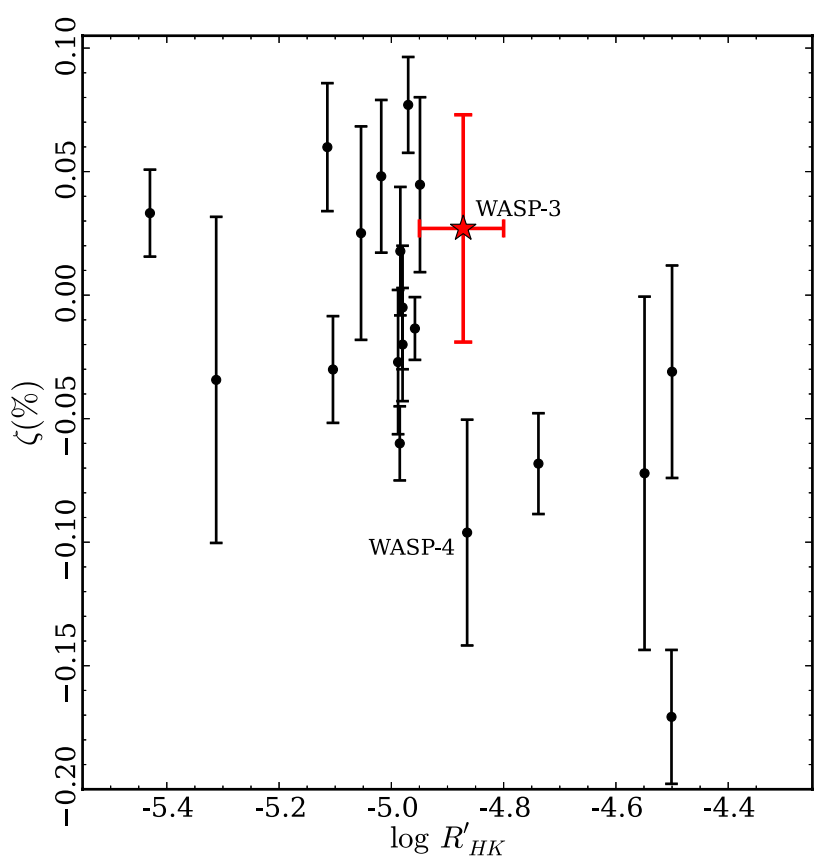

Figure 11. Stellar activity measure $\log R_{\mathrm{HK}}^{\prime}$ plotted against the empirical 3.6-4.5 $\mu \mathrm{m}$ slope measurement, $\zeta$. The labelled systems, WASP-3 and WASP-4, whilst having very similar host star activities, seem to display quite different vertical temperature structures. The $\log R_{\mathrm{HK}}^{\prime}$ error bar for WASP-3 reflects the range of activity values found for this object (Montalto et al. 2012).

inverted and non-inverted atmospheres (at $\log R_{\mathrm{HK}}^{\prime} \sim-4.9$ ). With $\log R_{\mathrm{HK}}^{\prime}=-4.872$ (Knutson et al. 2010), the WASP-3 system forms an interesting pair with WASP-4. This system has a very simi$\operatorname{lar} \log R_{\mathrm{HK}}^{\prime}$ value, but a shallower spectral slope than WASP-3b. This difference is also found in the model comparisons - WASP-4b is found to support either a weak inversion or no inversion at all (Beerer et al. 2011). Though the errors on the $\zeta$ values are large, it is still intriguing that the inversion/non-inversion cut-off with activity may not be as clear as the initial data suggested.

We note here that $\log R_{\mathrm{HK}}^{\prime}$ may not be well calibrated for WASP-3 due to its spectral type (late-F; Knutson et al. 2010). For stars of early spectral type, small amounts of emission in the $\mathrm{H}$ and $\mathrm{K}$ line cores can be difficult to detect due to the higher continuum flux. As such, we expect that any inaccuracy in the calibration of $\log R_{\mathrm{HK}}^{\prime}$ for WASP-3 would tend to underestimate the true value. A higher activity for WASP-3 would make for an even more intriguing comparison with WASP-4, given the proposed activity-inversion trend.

\section{CONCLUSION}

We have presented Spitzer observations of the WASP-3 system in three infrared bands, centred on 3.6, 4.5 and $8.0 \mu \mathrm{m}$ and have detected significant secondary eclipse signals from the planet WASP-3b in each band. The planet-to-star flux ratios derived from the eclipse depths were found to be $0.209_{-0.028}^{+0.040}, 0.282 \pm 0.012$ and $0.328_{-0.055}^{+0.086}$ percent, corresponding to brightness temperatures of $T_{3.6 \mu \mathrm{m}}=2280_{-150}^{+210} \mathrm{~K}, T_{4.5 \mu \mathrm{m}}=2400 \pm 80 \mathrm{~K}$ and $T_{8.0 \mu \mathrm{m}}=$ $2210_{-250}^{+390} \mathrm{~K}$.

We find that the strength of thermal emission from WASP$3 \mathrm{~b}$ suggests that the planet absorbs the incident stellar radiation 
efficiently and redistributes this energy to the night side of the planet inefficiently. The latter point is also heavily favoured when comparing the eclipse depths to the models of Fortney et al. (2008). These models also favour the presence of a temperature inversion in the atmosphere.

The WASP-3 system probes the cut-off of the suggested correlation between host star activity and the planetary $3.6-4.5 \mu \mathrm{m}$ spectral slope, and is found to have a slope consistent with other planets with temperature inversions. Another planet with a similar host star $\log R_{\mathrm{HK}}^{\prime}$ value, WASP-4b, has been found to have a weakly/non-inverted atmosphere. Since these objects appear to be on the cusp of the activity-inversion cut-off, it would be interesting to carry out monitoring of their $\log R_{\mathrm{HK}}^{\prime}$ and channel 2 eclipse depths. A detection of an anticorrelation between $\log R_{\mathrm{HK}}^{\prime}$ and the presence of an inversion would provide strong support for the destruction of a high-altitude absorber by UV irradiation as proposed by Knutson et al. (2010).

\section{ACKNOWLEDGEMENTS}

JWR acknowledges financial support from the Warwick Postgraduate Research Scholarship scheme. This work is based on observations made with the Spitzer Space Telescope, which is operated by the Jet Propulsion Laboratory, California Institute of Technology under a contract with NASA. Support for this work was provided by NASA through an award issued by JPL/Caltech. Astrophysics at Warwick is supported by an STFC grant.

\section{REFERENCES}

Agol E., Cowan N. B., Knutson H. A., Deming D., Steffen J. H., Henry G. W., Charbonneau D., 2010, ApJ, 721, 1861

Anderson D. R. et al., 2011, MNRAS, 416, 2108

Anderson D. R. et al., 2013, MNRAS, 430, 3422

Barman T. S., Hauschildt P. H., Allard F., 2005, ApJ, 632, 1132

Beerer I. M. et al., 2011, ApJ, 727, 23

Burrows A., Hubeny I., Sudarsky D., 2005, ApJ, 625, L135

Burrows A., Sudarsky D., Hubeny I., 2006, ApJ, 650, 1140

Burrows A., Budaj J., Hubeny I., 2008, ApJ, 678, 1436

Charbonneau D., Brown T. M., Latham D. W., Mayor M., 2000, ApJ, 529, L45

Charbonneau D., Brown T. M., Noyes R. W., Gilliland R. L., 2002, ApJ, 568,377

Charbonneau D. et al., 2005, ApJ, 626, 523

Charbonneau D., Knutson H. A., Barman T., Allen L. E., Mayor M., Megeath S. T., Queloz D., Udry S., 2008, ApJ, 686, 1341

Collier Cameron A. et al., 2007, MNRAS, 380, 1230

Cowan N. B., Agol E., 2011, ApJ, 729, 54

de Mooij E. J. W., de Kok R. J., Nefs S. V., Snellen I. A. G., 2011, A\&A, 528, A49

Deming D., Seager S., Richardson L. J., Harrington J., 2005, Nature, 434, 740
Deming D. et al., 2011, ApJ, 726, 95

Dhillon V. S. et al., 2007, MNRAS, 378, 825

Fortney J. J., Marley M. S., Lodders K., Saumon D., Freedman R., 2005, ApJ, 627, L69

Fortney J. J., Lodders K., Marley M. S., Freedman R. S., 2008, ApJ, 678, 1419

Fressin F., Knutson H. A., Charbonneau D., O’Donovan F. T., Burrows A., Deming D., Mandushev G., Spiegel D., 2010, ApJ, 711, 374

Gillon M. et al., 2007, A\&A, 471, L51

Hansen B. M. S., 2008, ApJS, 179, 484

Harrington J., Luszcz S., Seager S., Deming D., Richardson L. J., 2007, Nature, 447, 691

Hora J. L. et al., 2004, in Mather J. C., ed., Proc. SPIE Conf. Ser. Vol. 5487, Optical, Infrared, and Millimeter Space Telescopes. SPIE, Bellingham, p. 77

Hubeny I., Burrows A., Sudarsky D., 2003, ApJ, 594, 1011

Huitson C. M. et al., 2013, MNRAS, 434, 3252

Knutson H. A. et al., 2007, Nature, 447, 183

Knutson H. A., Charbonneau D., Allen L. E., Burrows A., Megeath S. T., 2008, ApJ, 673, 526

Knutson H. A., Howard A. W., Isaacson H., 2010, ApJ, 720, 1569

Kurucz R. L., 1979, ApJS, 40, 1

Lodders K., 2003, ApJ, 591, 1220

Machalek P., McCullough P. R., Burke C. J., Valenti J. A., Burrows A., Hora J. L., 2008, ApJ, 684, 1427

Maciejewski G. et al., 2010, MNRAS, 407, 2625

Madhusudhan N., Seager S., 2010, ApJ, 725, 261

Mathur S. et al., 2014, A\&A, 562, A124

Mazeh T. et al., 2000, ApJ, 532, L55

Moffat A. F. J., 1969, A\&A, 3, 455

Montalto M. et al., 2012, MNRAS, 427, 2757

Nascimbeni V. et al., 2013, A\&A, 549, A30

Pollacco D. et al., 2008, MNRAS, 385, 1576

Press W. H., Teukolsky S. A., Vetterling W. T., Flannery B. P., 1992, Numerical Recipes in FORTRAN: The Art of Scientific Computing. Cambridge Univ. Press, Cambridge

Sada P. V. et al., 2012, PASP, 124, 212

Schwarz G., 1978, Ann. Stat., 6, 461

Simpson E. K. et al., 2010, MNRAS, 405, 1867

Sing D. K. et al., 2013, MNRAS, 436, 2956

Southworth J., 2011, MNRAS, 417, 2166

Sudarsky D., Burrows A., Hubeny I., 2003, ApJ, 588, 1121

Todorov K., Deming D., Harrington J., Stevenson K. B., Bowman W. C., Nymeyer S., Fortney J. J., Bakos G. A., 2010, ApJ, 708, 498

Todorov K. O. et al., 2012, ApJ, 746, 111

Todorov K. O. et al., 2013, ApJ, 770, 102

Tripathi A. et al., 2010, ApJ, 715, 421

Wheatley P. J. et al., 2010, preprint (arXiv:1004.0836)

Zahnle K., Marley M. S., Freedman R. S., Lodders K., Fortney J. J., 2009, ApJ, 701, L20

Zhao M., Milburn J., Barman T., Hinkley S., Swain M. R., Wright J., Monnier J. D., 2012, ApJ, 748, L8

This paper has been typeset from a $\mathrm{T}_{\mathrm{E}} \mathrm{X} / \mathrm{L} \mathrm{T}_{\mathrm{E}} \mathrm{X}$ file prepared by the author. 